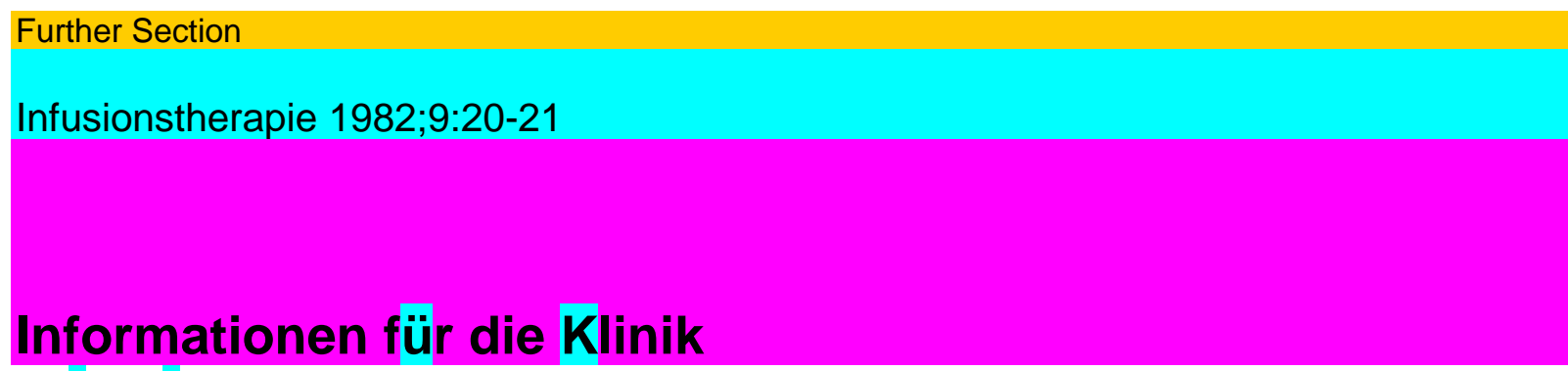

\title{
Inncre Mcdizin
}

Implantierbare Insulinpumpe In Forschung befindet sich ein implantierba-res und programmierbares Insulin-Dosier-gerät zur Insulinlangzeitzufuhr. Aufbauend auf das extern zu tragende Gerät Promedos El hat Siemens ein Dosiergerät entwickelt, das in einem Herzschrittmachergehäuse aus Titan untergebracht ist und gefüllt $170 \mathrm{~g}$ wiegt. Der Speicher, der für etwa 4 Wochen reicht, kann über eine Injektionsnadel durch die Haut nachgefüllt werden. Zwei Implantationsorte werden gegenwärtig un-tersucht. Bei der Implantation auf das Bauchfell im Unterbauchfettgewebe gelangt das Insulin über sehr dünne, mit Silikon-gummi überzogene Polyaethylenkatheter in den Bauchraum, wo es resorbiert wird und in die Blutbahn gelangt. Die Implantation im oberen Thoraxbereich erlaubt die pro-thrahierte Zufuhr des Insulins über einen Katheter in die Vena subclavia. Die vorge-nommene Implantationstechnik erfolgt analog der Schrittmacherimplantation und stellt daher kein technisches Problem dar. Das Gerät kann auf eine konstante Grundrate programmiert werden, welcher bedarfsge-recht zu den Mahlzeiten eine zusätzliche Insulindosis zugesetzt wird. Dies erfolgt über ein externes Programmiergerat von Taschenrechnergröße. In Zusammenarbeit mit Hoechst wurde auch ein spezielles Insulin entwickelt, das den für Langzeitdosiergeräte erforderlichen Ansprüchen gerecht wird: Es ist neutral, hochkonzentriert, bei Körpertemperatur wochenlang stabil, wird durch Schütteln nicht inaktiviert und zeigt keine Unverträg-lichkeiten mit dem Pumpenmaterial und den Kathetern. Mit dieser Entwicklung können die Patienten ähnlich wie Schrittmacher-patienten unbehindert ihre Arbeit und ihren Freizeitbeschäftigungen nachgehen. Um-fangrèichen klinischen Studien wird es vor-behalten sein, Vorteile und Risiken zu erfas-sen, so daß der klinische Routineeinsatz und die Serienreife dieses Gerätes noch einige Jahre anstehen werden.

Radiologie

Risikoarme Angiographie durch digitale Substraktionsangiographie (D VSA)

Neu entwickelte Mikroelektronik mit digita-ler Bildverarbeitung ist auf dem Weg, die klassischen Techniken der Angiographie er-heblich zu bereichern. Mit dem Angiotron hat Siemens ein Gerät geschaffen, das mit-tels des Prinzips der Substraktionstechnik es erlaubt, mit geringsten Kontrastmittelkon-zentrationen Gefäßdarstellungen zu errei-chen.

Wesentlicher Vorteil ist es, das Kontrastmit-tel auch intravenös zuführen und damit die bekannten Risiken senken zu können. Dies erlaubt eine entscheidende Erweiterung der diagnostischen Indikationen, insbesondere bei Patienten mit erhöhtem Risiko. Die Angiographie kann dadurch in vielen Fallen ambulant durchgeführt werden, was zu er-heblicher Kostenersparnis führen dürfte. Die Substraktionstechnik erlaubt es, Bilder einer angiographischen Aufnahme verstärkt sichtbar zu machen, indem durch Substrak-tion der unveränderte Hintergrund der Röntgenaufnahmen eleminiert wird. Die entscheidende Voraussetzung für die Qualität dieses Verfahrens ist die Exaktheit der Deckungsgleichheit der kontrastmittel-gefüllten Gefäße mit den von ihnen zu sub-strahierenden Leeraufnahmen. Echtzeitdar-stellungen von Substraktionsbildern waren bislang aufgrund des Zeitaufwandes durch photographische und elektronische Sub-straktionsmethoden nicht zu erreichen. Analoge 
Videosignale aus einer Röntgen-Bild-Verstärker-Fernseh-Kette werden im Angiothron in digitale Signale einer Bild-matrix mit 512x512 Bildpunkten, die je-weils in ein 12-bit-Wort verschlüsselt sind, umgewandelt. Von den gespeicherten Digi-talwerten des Leerbildes werden die Digitalwerte des Füllungsbildes substrahiert. Aus der Differenz der Digitalwerte entsteht ein analoges Videosignal, das auf dem Fernseh-monitor als Substraktionsbild erscheint. Eine EchtzeitDarstellung von angiographischen Funktionsabläufen entsteht dadurch, daß durch die Darstellung von 50 Bildern pro Sekunde der Vorgang extrem schnell abläuft. Dadurch erscheinen auf dem Monitor Substraktionsbilder, die normalen Rönt-genFernsehdurchleuchtungen vergleichbar sind. Die Darstellung auch geringster Kontrastmittelkonzentrationen ist durch die außerordentliche Empfindlichkeit des An-giothrons möglich. Dies ermöglicht geringe Kontrastmittelkonzentrationen mit der Möglichkeit der intravenösen Zufuhr und vermindert daher den Kreis der Patienten mit Kontraindikationen. Das gilt insbesondere für Patienten mit Niereninsuffizienz, bei Patienten, bei denen lange arterielle Kontrastmittelinjektionen zu kritischer Sauerstoffuntersättigung führen könnte oder bei denen die erheblichen Risiken der

selektiven Arteriographie der Halsarterie umgangen werden sollte. Das Angiothron eröffnet somit eine Methode, die über die intravenöse Angiographie risikoärmer, bes-ser tolerabel und aufgrund der ambulanten Durchführbarkeit erheblich kostensparen-der ist. Die geringere Detailauflösung im Vergleich zur konventionellen Angiographie tritt angesichts des geringeren Untersu-chungsrisikos und der erheblich verminder-ten Belastung des Patienten in den Hintergrund.

Umfangreiche klinische Studien werden je-doch erforderlich sein, diese Erwartungen zu bestätigen.

Labor

Saure Phosphatase -neuer enzymatischer Monotest Die Aktivitätsbestimmung der sauren Phosphatase nimmt einen festen Platz in der Diagnostik der Inneren Medizin, der Urolo-gie und der Gynäkologie ein. Eine Reihe von Erkrankungen führt zu einem Anstieg der gesamtsauren Phosphatase und - oder der prostataspezifischen Phosphatase beim Erwachsenen, wie z.B. Prostatakarzinom, bei dem die Erhöhung der Serumaktivität am ausgeprägtesten ist, Adenomyomatose der Prostata, Prostatitis, Knochenmarkserkrankungen, wie z.B. osteolytisches Sarkom, M. Paget, Blut-erkrankungen wie Thrombozytämie, Mega-loblastische Anämie, Myelosen, M. Gau-cher und das metastasierende Mammakarzi-nom. Die vorliegende neue Methode ermöglicht eine kinetische Messung, die über die Extensionsdifferenz pro Minute photo-metrisch die Aktivität der sauren Phosphatase ermöglicht. Neue Aspekte und Vorteile dieser Methode sind die schnelle Durchführbarkeit, die Automatisierbarkeit und die einfache Handhabung durch ein sogenann-tes Tablettenkonzept. Dem Prinzip dieses neuen Monotests von Boehringer/Mannheim liegt die von Hillmann beschriebene Methode zugrunde [Hillmann, G., Z. klin. Chem. und Klin. Biochem. 9, 273,1971]. Dabei setzt die saure Phosphatase 1-Naphtylphosphat enzymatisch in 1 Naphtol um, das durch Kopplung mit diazotierten Aminochlortoluol zu einem Azofarbstoff umgewandelt wird. Die Bildung des Farb-stoffs wird bei Hg 405 nm kontinuierlich ge-messen und stellt ein Maß für die Enzym-aktivität dar. Bis zu einer Extinktionsdiffe-renz von $\mathrm{E} / \mathrm{min}=0,1$ oder $74 \mathrm{U} / \mathrm{l}$ besteht eine Liniarität. Der Test ist bei RaumtempeInformationen für die Klinik 21 
ratur 24 Stunden und im Kühlschrank 48 Stunden haltbar. Keinerlei Einfluß auf den Test zeigten 37 verschiedene und häufig ein-gesetzte Pharmaka.

Neben der manuellen Durchführung, die einfach und rasch in der technischen Hand-habung ist, ist die Methode für die Analy-senautomaten ACP 5040, Centrifilchem, Causac und Cobar Bio problemlos und defi-niert anwendbar.

Die handelsübliche Packung reicht für 20 Testbestimmungen und ist bei Boehringer/ Mannheim als monotestsaure Phosphatase Best.-Nr. 256676 erhältlich.

Kongresse

Die Bundesärztekammer veranstaltete vom 13. bis 16. Januar 1982 ein interdisziplinäres Forum »Fortschritt und Fortbildung in der Medizin«, das Dr. Karsten Vilmar, Präsi-dent der Bundesärztekammer und des Deut-schen Ärztetages eröffnete. Stationäre Behandlung der Virushepatitis in der Regel nicht erforderlich. Bei Virushepatitis bedürfen lediglich Risikopatienten der stationären Behandlung. Prof. Dr. J.C. Bode stellte fest, daß die Infektionsgefahr sowohl bei Virus A als auch bei Virus B generell überschätzt wird. Bei Auftreten des Ikterus sei die Hepatitis A in der Regel schon nicht mehr anstek-kend. Die Ansteckungsgefahr bei Hepatitis B besteht wohl länger - die Infektionsgefahr sei jedoch für das Klinikpersonal höher als für die Angehörigen zu Hause, da die Übertragung vornehmlich über das Blut er-folgt. Für die früher in vielen Kliniken durchgeführte Infusionsbehandlung mit Fructose gibt es keine Indikation, da in einer großen Zahl von Studien gezeigt wer-den konnte, daß der Heilungsverlauf durch Fructose nicht beeinflußt wird. Die anwesenden Klinikärzte und niederge-lassenen Ärzte waren sich daher einig, daß die komplikationslose Virushepatitis, wie auch in anderen europäischen Ländern zu Hause behandelt werden kann, da die Behandlung in der Klinik bei komplikations-losem Verlauf keine Vorteile erbringt.

Ulcera des Ösophagus durch Tabletten

Besonders kurz vor dem Schlafengehen ein-genommene Tabletten können zu lange im Ösophagus verweilen und dort zu Irritatio-nen der Schleimhaut bis hin zu Ulcera füh-ren. Prof. Dr. M. Wienbeck, Düsseldorf, emp-fahl daher, die Patienten anzuhalten, bei Tabletteneinnahme im Bett ausreichend Flüs-sigkeit nachzutrinken.

Kombinierte Strahlentherapie und Hyperthermie bei schweren Tumor-Schmerzen Durch zweimal wöchentliche Strahlenbe-handlung schmerzhafter Tumorregionen mit nachfolgender lokaler Hyperthermie von 42-48 Grad C für eine Stunde konnten Tumorschmerzen wirksamer bekämpft werden und eine deutlichere Tumorrückbildung bewirken als alleinige Strahlenbehandlung, berichtete Prof. Dr. E. Scherer, Essen Verbesserte Prognose bei Leukämien im Kindesalter Aufgrund der Erfolge in der chemotherapeutischen Behandlung kindlicher Leukämien kommt der Frühdiagnose eine wichtige Bedeutung zu, führte Prof. Dr. G. Schellong aus und wies auf die Erfordernis der ärztli-chen Aufklärung der Eltern hin. Dabei sei aufgrund der weitaus besseren Prognose im Vergleich zu früher eine pessimistische Grundhaltung nicht am Platze, da durchaus die Hoffnung auf endgültige Heilung be-stehe.

Wichtige Kongreßtermine: ESPEN 82: In Wien vom 26.-29.9.82. Der 4. Kongreß der European Society of Parenteral and Enteral Nutrition findet vom

26.-29.9.82 in Wien statt. Unter der Lei-tung von Prof. Dr. E. Deutsch sind folgende Themenschwerpunkte vorgesehen: Ernäh-rung bei Herzkrankheiten, bei akutem Nierenversagen, bei Leberinsuffizienz, bei aku-ter Pankreatitis, bei Tumoren, bei entzündli-chen Darmerkrankungen. Weitere Themen behandeln Ernährungsprobleme von Früh-geborenen und Kindern sowie Langzeit-parenterale Ernährung und endokrinologi-sche Aspekte. 
Nähere Auskunft erfolgt über Doz. Dr. G. Kleinberger, Congress Secretariat, P.O. Box 9, A1095 Wien und Tel. 0222/526839.

Allgemeines

Paul-Martini-Preis 1982 Der Paul-Martini-Preis für Arbeiten, die zur Weiterentwicklung wissenschaftlicher Me-thoden der Beurteilung klinisch-pharmako-logischer und therapeutischer Maßnahmen beitragen, ist für 1982 wieder international ausgeschrieben worden. Die von der Paul-Martini-Stiftung der Med. pharmazeutischen Studiengesellschaft e.V (Mainz) verliehene Auszeichnung ist mit DM 20000 dotiert. Die Arbeiten sollen in ófacher Ausfertigung in deutscher oder eng-lischer Sprache an die Med. pharmazeuti-sche Studiengesellschaft e.V, Postfach 3048, D-6500 Mainz, gerichtet werden. Die Arbeiten, die bereits publiziert, aber nicht alter als 3 Jahre sein dürfen, sollen in sich abgeschlossen sein. Einsendeschluß ist der 30.4.1982. Jede Arbeit ist mit einem Kennwort zu versehen und darf den Namen des Verfassers nicht enthalten. In einem se-paraten verschlossenen Umschlag sind Kennwort der Arbeit und exakte Perso-nalien anzugeben.

Verantwortlich für die Zusammenstellung: Dr. med. U. Sprandel, München 